\title{
ON THE DIFFERENCE BETWEEN DYNAMIC CHROMATIC NUMBER AND CHROMATIC NUMBER OF GRAPHS WITHOUT SOME SUBGRAPHS
}

\section{BO GAO ${ }^{1}$, LEI SUN ${ }^{2}$, HUIMIN SONG ${ }^{3}$ and HONG-JIAN LAI ${ }^{4}$}

${ }^{1}$ School of Information Sciences and Engineering

Shandong Normal University

Jinan 250014

P. R. China

e-mail: b.gao@163.com

2Department of Mathematics

Shandong Normal University

Jinan 250014

P. R. China

e-mail: lsun89@163.com

${ }^{3}$ School of Mathematics and Statistics

Shandong University at Weihai

Weihai 264209

P. R. China

e-mail: hmsong@sdu.edu

${ }^{4}$ Department of Mathematics

West Virginia University

Morgantown, WV 26506-6310

USA

e-mail: hjlai@wvu.edu

2010 Mathematics Subject Classification: 05C15.

Keywords and phrases: chromatic number, dynamic chromatic number, $K_{1,3}$-free graph,

$K_{1,4}$-free graph, even cycle.

This work is supported by the National Natural Science Foundation of China (Grant No. 11271365).

Received May 9, 2015 


\begin{abstract}
For integers $k, r>0$, a $(k, r)$-colouring of a graph $G$ is a proper colouring on the vertices of $G$ by $k$ colours such that every vertex $v$ of degree $d(v)$ is adjacent to vertices with at least $\min \{d(v), r\}$ different colours. The dynamic chromatic number, denoted by $\chi_{2}(G)$, is the smallest integer $k$ for which a graph $G$ has a $(k, 2)$-colouring. In this paper, we prove a sufficient condition for a $K_{1,3}$-free graph $G$ with $\chi_{2}(G)=\chi(G)$. Also, we give some upper bounds for $\chi_{2}(G)-\chi(G)$ of $K_{1,4}$-free graphs and graphs without even cycles.
\end{abstract}

\title{
1. Introduction
}

All graphs in this paper are finite, undirected, and simple. We follow the notation and terminology of [2]. Thus for a graph $G, \Delta(G)$ and $\chi(G)$ denote the maximum degree and the chromatic number of $G$. If vertices $u$ and $v$ are connected in $G$, the distance between $u$ and $v$ in $G$, denoted by $d_{G}(u, v)$, is the length of a shortest $(u, v)$-path in $G$. For $u \in V(G)$, let $N_{i}(u)=\left\{v: v \in V(G), d_{G}(u, v)=i\right.$. We use the symbol $N(v)$ to denote $N_{1}(v)$, and $d(v)=|N(v)|$.

For an integer $k>0$, a proper $k$-colouring of a graph $G$ is a map $c: V(G) \mapsto\{1,2, \cdots, k\}$ such that if $u, v \in V(G)$ are adjacent vertices in $G$, then $c(u) \neq c(v)$. The smallest $k$ such that $G$ has a proper $k$-colouring is the chromatic number of $G$, denoted by $\chi(G)$. A proper vertex $k$-colouring of a graph $G$ is called dynamic if for every vertex $v$ with degree at least 2 , the neighbours of $v$ receive at least two different colours. The smallest integer $k$ such that $G$ has a dynamic $k$-colouring is called the dynamic chromatic number of $G$ and denoted by $\chi_{2}(G)$.

The concept of dynamic colouring of graphs was first introduced in [9] and [6]. Later in [5], it is called conditional colouring. Lately, it has been studied extensively by several authors in [3, 4, 6, 7]. Obviously, $\chi(G) \leq \chi_{2}(G)$. It was shown in [8] that the difference between the 
chromatic number and the dynamic chromatic number can be arbitrarily large. However, it was conjectured in [9] that for regular graphs the difference is at most 2. In [7], it has been proved that the computational complexity of $\chi_{2}(G)$ for a 3-regular graphs is an NP-complete problem. It is an interesting problem to investigate the optimal upper bound for $\chi_{2}(G)-\chi(G)$. In this paper, we present some bounds for $\chi_{2}(G)-\chi(G)$ of graphs without some subgraphs.

\section{2. $K_{1,3}$-free Graphs and $K_{1,4}$-free Graphs}

A graph $G$ is $K_{1, r}$-free if $G$ does not have an induced subgraphs isomorphic to $K_{1, r}$. In [5], it was proved that if $G$ is a connected and $K_{1,3}$-free, then $\chi_{2}(G) \leq \chi(G)+2$, and the equality holds if and only if $G$ is a cycle of length 5 or of even length not a multiple of 3 . We will prove a sufficient condition for a $K_{1,3}$-free graph $G$ with $\chi_{2}(G)=\chi(G)$.

Let $c$ be a proper vertex colouring of a graph $G$, we denote the vertex set which receives the colour $i$ by $c^{-1}(i)$. We denote the set of colours which appear in the vertex set $V$ by $c(V)$. If for a vertex $v$ with degree at least $2,|c(N(v))|=1$, then $v$ is called a bad vertex, otherwise, it is called a good vertex. If a vertex $v$ satisfies $d(u)=2, N(u)=\left\{v_{1}, v_{2}\right\}$ and $d\left(v_{1}\right) \geq 3, d\left(v_{2}\right) \geq 3$, we call $u$ the unique middle vertex.

Lemma 2.1. Let $G$ be a graph.

(i) If $G[N(u)]$ has an edge, then $u$ is good.

(ii) If $G$ is $K_{1, r}$-free, then every vertices of degree at least $r$ is good.

The proof of Lemma 2.1 is trivial. 
Theorem 2.2. Let $G$ be a $K_{1,3}$-free graph with $\chi(G) \geq 4$, and there is no unique middle vertex in $G$, then $\chi_{2}(G)=\chi(G)$.

Proof. Let $G$ be a $K_{1,3}$-free graph with $\chi(G) \geq 4$, and there is no unique middle vertex in $G$. Suppose $G$ has a proper vertex colouring $c: V(G) \mapsto\{1,2, \ldots, \chi(G)\}$.

Because $G$ is a $K_{1,3}$-free graph, then by Lemma 2.1 all the vertices we should consider are the vertices with degree 2 .

Suppose $d(u)=2$.

Case 1. $u$ is contained in a triangle. Then by Lemma 2.1, it is a good vertex.

Case 2. $u$ is not in any triangle of $G$, then $u$ is in a deduced subgraph $P$ of $G$ which is a path, and the degree of the end point of $P$ is not 2 .

Suppose the endpoint of $P$ is $v_{1}, v_{2}, c\left(v_{1}\right)=c_{1}, c\left(v_{2}\right)=c_{2}$. Because there is no unique middle point vertex in $G$, the inner vertices of $P$ are more than two.

Subcase 2.1. There are two inner vertices in $P$ since $\chi(G) \geq 4$, then there are two colours $c_{3}, c_{4}$, which are different from $c_{1}, c_{2}$ can be used at these vertices, then we have done.

Subcase 2.2. There are $3 n\left(n \in N^{+}\right)$inner vertices in $P$, we can colour the vertices in $P$ as

$$
c_{1} \underbrace{c_{2} c_{3} c_{4} c_{2} c_{3} c_{4} \cdots c_{2} c_{3} c_{4}}_{3 n} c_{2} .
$$

Subcase 2.3. There are $3 n+1\left(n \in N^{+}\right)$inner vertices in $P$, we can colour the vertices in $P$ as

$$
c_{1} \underbrace{c_{2} c_{3} c_{4} c_{2} c_{3} c_{4} \cdots c_{2} c_{3} c_{4}}_{3 n} c_{1} c_{2}
$$


Subcase 2.4. There are $3 n+2\left(n \in N^{+}\right)$inner vertices in $P$, we can colour the vertices in $P$ as

$$
c_{1} \underbrace{c_{2} c_{3} c_{4} c_{2} c_{3} c_{4} \cdots c_{2} c_{3} c_{4}}_{3 n} c_{1} c_{3} c_{2} .
$$

Finally, we keep the colours of all the other vertices, then we have a dynamic colouring of $G$ with $\chi(G)$ colours.

The condition that there is no unique middle vertex in $G$ is necessary. In fact, we can find a graph with $\chi(G) \geq 4$, which contains a unique middle vertex satisfying $\chi_{2}(G)=\chi(G)+1$, see Figure 1 .

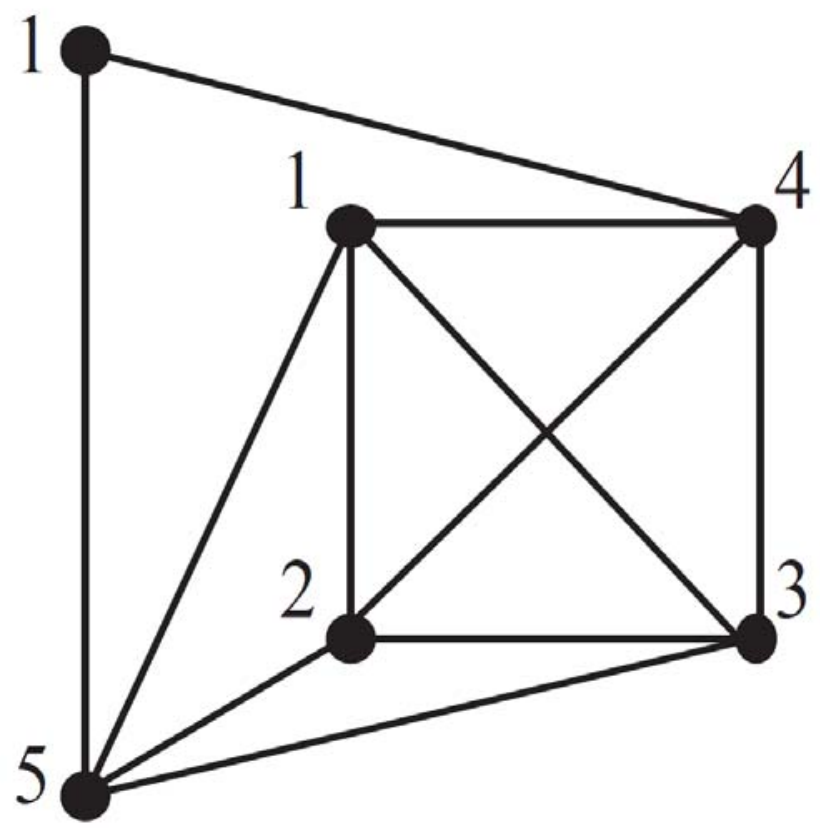

Figure 1. Graph for the note of Theorem 2.2.

Lemma 2.3 ([6]). For a connected graph $G$ if $\Delta(G) \leq 3$, then $\chi_{2}(G) \leq 4$ unless $G=C_{5}$, in which case $\chi_{2}\left(C_{5}\right)=5$, and if $\Delta(G) \geq 4$, then $\chi_{2}(G) \leq \Delta(G)+1$. 
Theorem 2.4. Let $G$ be a $K_{1,4}$-free graph, then $\chi_{2}(G) \leq 2 \chi(G)$.

Proof. Let $G$ be a counterexample with fewest number of vertices.

If $\chi(G)=1$, it is trivial. If $\chi(G)=2, G$ is $K_{1,4}$-free, then $\Delta(G) \leq 3$.

By Lemma 2.3, $\chi_{2}(G) \leq \Delta+1=4$.

Let $\chi(G) \geq 3$. Let $c^{\prime}: V(G) \mapsto\{1,2, \cdots, \chi(G)\}$ is a proper colouring of $G$.

Because $G$ is a counterexample and $K_{1,4}$-free, then there is a bad vertex $u$ in $G$ and $d(u) \leq 3$.

Let $v$ is a neighbour of $u$, then every colour received by vertices in $N(v) \backslash\{u\}$ can not appears more than twice. Otherwise, there will be a $K_{1,4}$, so $d(v) \leq(\chi(G)-1) \times 2+1=2 \chi(G)-1$.

Let $\quad c: V(G) \backslash\{u\} \mapsto\{1,2, \cdots, 2 \chi(G)\}$ is a dynamic colouring of $G-u$. Because $G$ is a counterexample, then $|c(N(u))|=1$. Without loss of generality, let this colour to be 1 . Let $v$ to be a neighbour of $u$. Because $d(v) \leq 2 \chi(G)-1$, then there is at least one colour missing in $N(v) \backslash\{u\}$. Without loss of generality, let this colour to be $2 \chi(G)$.

Case 1. For any vertex $w$ in $N(v) \backslash\{u\}$, there is only one colour assigned to vertices in $N(w) \backslash\{v\}$.

Because $|N(v) \backslash\{u\}| \leq 2 \chi(G)-2$, then there is one colour $i(i \neq 1)$ missing in $N_{2}(v)$. If $i$ does not appear in $N(v) \backslash\{u\}$, let $\widetilde{c}(v)=i$. If $i$ appears in $N(v) \backslash\{u\}$, suppose $c\left(w_{0}\right)=i$. Because $c$ is a 2-hued colouring of $G-u$, then the colour 1 does not appear in $N_{2}(v)$. Let $\tilde{c}\left(w_{0}\right)=1, \tilde{c}(v)=i$.

Case 2. There is a vertex $w_{i_{0}}$ in $N(v) \backslash\{u\}$ so that there are at least two colours appearing in $N\left(w_{i_{0}}\right) \backslash\{v\}$.

Because $G$ is a counterexample and the colour $2 \chi(G)$ does not appear in $N(v) \backslash\{u\}$, then there is a vertex $w_{i_{1}}$ in $N(v)$ so that the vertex in $N\left(w_{i_{1}}\right) \backslash\{v\}$ receive only one colour $2 \chi(G)$. If there is no vertex $w_{i_{2}}$ in 
$N(v) \backslash\{u\}$ so that there is only one colour $c\left(w_{i_{1}}\right)$ in $c\left(N\left(w_{i_{2}}\right) \backslash\{v\}\right)$. Let $\tilde{c}\left(w_{i_{1}}\right)=1, \tilde{c}(v)=c\left(w_{i_{1}}\right)$. If such vertex $w_{i_{2}}$ exists, we can get a vertex $w_{i_{3}}$ so that there is only one colour $c\left(w_{i_{2}}\right)$ appears in $c\left(N\left(w_{i_{3}}\right) \backslash\{v\}\right)$. Continuing this procedure, we can stop at a vertex $w_{i_{j}} \in N(v) \backslash\{u\}$ such that there is no vertex $w_{i_{j+1}}$ in $N(v) \backslash\{u\}$ having the property that there is only one colour $c\left(w_{i_{j}}\right)$ in $c\left(N\left(w_{i_{j+1}}\right) \backslash\{v\}\right)$. Let $\widetilde{c}\left(w_{i_{j}}\right)=1, \widetilde{c}(v)=c\left(w_{i_{j}}\right)$. For any vertex $w \in N(v) \backslash\{u\}$ satisfying $c(w)=c\left(w_{i_{j}}\right)$, let $\tilde{c}(w)=1$.

At last, let $\tilde{c}(v)=c(v)$ for all the other vertices in $G-u$. Because $d(u) \leq 3$, we can easily colour the vertex $u$ and get $\tilde{c}$, which is a dynamic colouring of $G$ with $2 \chi(G)$ colours, a contradiction.

The upper bound in the Theorem 2.4 is best impossible. We can construct a $K_{1,4}$-free graph $G$ with $\chi_{2}(G)=2 \chi(G)$ as following. Let $G^{\prime}$ be a complete $r$-partite graph, $G^{\prime}$ has 2 nonadjacent vertices in $i$-th $(1 \leq i \leq r)$ vertex class and contains all edges joining vertices in distinct class. At last, we get $G$ from $G^{\prime}$ by joining the two vertices in $i$-th $(1 \leq i \leq r)$ vertex class by a path of length two. It is easy to see that $\chi(G)=r$ and $\chi_{2}(G)=2 r$. An example graph $G$ with $\chi(G)=4$ and $\chi_{2}(G)=8$ is shown in Figure 2 . 


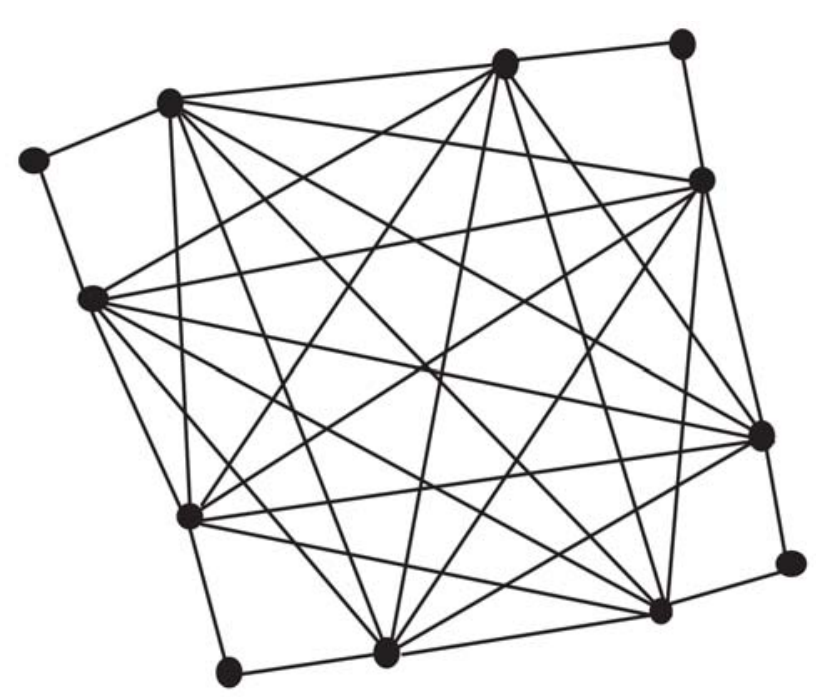

Figure 2. Graph for the note of Theorem 2.4.

\section{The Graphs Without Subgraphs $C_{2 n}\left(n \geq 2, n \in N^{+}\right)$}

Theorem 3.1. If $G$ does not have a subgraph $C_{2 n}\left(n \geq 2, n \in N^{+}\right)$, then $\chi_{2}(G) \leq 2 \chi(G)$.

Proof. Let $c: V(G) \mapsto\{1,2, \cdots, \chi(G)\}$ to be a proper colouring of $G$ such that every vertex of colour $i$ has at least a neighbour of colour $j$, for every $j<i$. Clearly, all the bad vertices must be in $c^{-1}(1)$ and $c^{-1}(2)$.

Let $F_{1}$ to be a subgraph of $G$ induced by $V^{1}$ and the neighbours of $V^{1}, V^{1}=\{v \mid c(v)=1, v$ is a bad vertex and the neighbours of $v$ are all in $\left.c^{-1}(2)\right\}$. Let $F_{2}$ to be a subgraph of $G$ induced by $V^{2}$ and the neighbours of $V^{2}, V^{2}=\{v \mid c(v)=2, v$ is a bad vertex and the neighbours of $v$ are all in $\left.c^{-1}(1)\right\}$. Let $F=F_{1} \bigcup F_{2}$, then $F$ is a bipartite graph. We call the vertex set of $F$ which have the colour $1 V_{1}$, and we call the other vertex 
set of $F V_{2}$. Denote $V_{i}=\left\{v \mid v \in c^{-1}(1), v\right.$ is a bad vertex, the neighbour of $v$ is in $\left.c^{-1}(i)\right\}, 3 \leq i \leq \chi(G)$. We call the bipartite graph induced by $V_{i}$ and the neighbours of $V_{i} F_{i}, 3 \leq i \leq \chi(G)$.

We construct graphs $G_{i}\left(V_{i}, E_{i}\right), 1 \leq i \leq \chi(G)$ by the algorithm as following:

Step 0. Let $V:=V_{i}, E_{i}:=\varnothing$.

Step 1. Choose a vertex $v$ from $V$, then choose two vertices $v^{\prime}, v^{\prime \prime}$ from $N(v)$. Let $e:=\left(v^{\prime}, v^{\prime \prime}\right)$.

Step 2. Let $E_{i}:=E_{i} \cup\{e\}, V:=V \backslash\{v\}$. If $V \neq \varnothing$, return Step 1 .

Step 3. Let $G_{i}:=G\left(N\left(V_{i}\right), E_{i}\right)$.

For every $1 \leq i \leq \chi(G), G_{i}\left(N\left(V_{i}\right), E_{i}\right)$ is a bipartite graph. Otherwise, there is an odd cycle in $G_{i}$ and there is an even cycle in $G$ by the denote of $E_{i}$. We change the colour of one part of $G_{i}$ to a new colour $\chi(G)+i(1 \leq i \leq \chi(G))$ and keep the colours of all the other vertices. We eventually get a dynamic colouring of $G$ with $2 \chi(G)$ colours.

\section{References}

[1] Meysam Alishahi, On the dynamic coloring of graphs, Discrete Applied Mathematics 159 (2011), 152-156.

[2] J. A. Bondy and U. S. R. Murty, Graph Theory with Applications, Elsevier North Holland, 1981.

[3] C. Ding, S. Fan and H.-J. Lai, Upper bound on conditional chromatic number of graphs, J. of Jinan University 29 (2008), 7-14.

[4] Louis Esperet, Dynamic list coloring of bipartite graphs, Discrete Applied Mathematics 158 (2010), 1963-1965.

[5] H.-J. Lai, J. Lin, B. Montgomery, Z. Tao and S. Fan, Conditional colorings of graphs, Discrete Mathematics 306 (2006), 1997-2004. 
[6] H.-J. Lai, B. Montgomery and H. Poon, Upper bounds of dynamic chromatic number, Ars Combinatoria 68 (2003), 193-201.

[7] Xueliang Li, Xiangmei Yao, Wenli Zhou and Hajo Broersma, Complexity of conditional colorability of graphs, Applied Mathematics Letter 22 (2009), 320-324.

[8] Yue Lin, Upper Bounds of Conditional Chromatics Number, Master Thesis, Jinan University, 2008.

[9] B. Montgomery, Dynamic Coloring of Graphs, Ph.D. Thesis, West Virginia University, 2001. 